\title{
DIFFERENT ASSEMBLY OF TYPE IV COLLAGEN ON HYDROPHILIC AND HYDROPHOBIC SUBSTRATA ALTERS ENDOTHELIAL CELLS INTERACTION
}

\author{
N. Miranda Coelho ${ }^{1,2}$, C. González-García ${ }^{3}$, J. A. Planell ${ }^{1,2}$, M. Salmerón-Sánchez ${ }^{3,4,5}$, and G. Altankov ${ }^{1,4,6 *}$ \\ ${ }^{1}$ Institut de Bioenginyeria de Catalunya, Barcelona, Spain \\ ${ }^{2}$ Universitat Politècnica de Catalunya (UPC), Barcelona, Spain \\ ${ }^{3}$ Center for Biomaterials and Tissue Engineering, Universidad Politécnica de Valencia, 46022 Valencia, Spain \\ ${ }^{4}$ Networking Research Center on Bioengineering, Biomaterials and Nanomedicine \\ (CIBER-BBN), Valencia, Spain \\ ${ }^{5}$ Regenerative Medicine Unit, Centro de Investigación Príncipe Felipe, Autopista del Saler 16, 46013 Valencia, \\ Spain \\ ${ }^{6}$ ICREA (Institució Catalana de Recerca i Estudis Avançats), Barcelona, Spain
}

\begin{abstract}
Considering the structural role of type IV collagen $(\mathrm{Col}$ IV) in the assembly of the basement membrane (BM) and the perspective of mimicking its organization for vascular tissue engineering purposes, we studied the adsorption pattern of this protein on model hydrophilic (clean glass) and hydrophobic trichloro(octadecyl)silane (ODS) surfaces known to strongly affect the behavior of other matrix proteins. The amount of fluorescently labeled Col IV was quantified showing saturation of the surface for concentration of the adsorbing solution of about $50 \mu \mathrm{g} / \mathrm{ml}$, but with approximately twice more adsorbed protein on ODS. AFM studies revealed a fine - nearly single molecular size - network arrangement of Col IV on hydrophilic glass, which turns into a prominent and growing polygonal network consisting of molecular aggregates on hydrophobic ODS. The protein layer forms within minutes in a concentration-dependent manner. We further found that human umbilical vein endothelial cells (HUVEC) attach less efficiently to the aggregated Col IV (on ODS), as judged by the significantly altered cell spreading, focal adhesions formation and the development of actin cytoskeleton. Conversely, the immunofluorescence studies for integrins revealed that the fine Col IV network formed on hydrophilic substrata is better recognized by the cells via both $\alpha 1$ and $\alpha 2$ heterodimers which support cellular interaction, apart from these on hydrophobic ODS where almost no clustering of integrins was observed.
\end{abstract}

Keywords: Collagen type IV, adsorption, assembly, hydrophilic, hydrophobic, surfaces.

\footnotetext{
*Address for correspondence:

G. Altankov

ICREA - Molecular Dynamics

Feixa Llarga Pavelo Govern Planta 1 No 1112 ,

Bellvitge Barcelona Hospitalet de Llobregat

08907 Barcelona, Spain
}

Telephone Number:

FAX Number:

E-mail: george.altankov@icrea.es

\section{Introduction}

The initial cell-biomaterials interaction mimics to a certain extent the natural communication of cells with the extracellular matrix (ECM); it starts with the adsorption of soluble matrix proteins from the surrounding medium followed by cell adhesion, spreading and polarization (Grinnell and Feld, 1982; Griffith and Naughton, 2002; Sipe, 2002). In some cases however, less soluble ECM proteins such as collagens or laminins also associate with the biomaterial surface eliciting distinct cellular responses. In this study we were particularly interested in the behavior of adsorbed type IV collagen (Col IV) - a unique multifunctional matrix protein that plays a crucial role in the organization of the basement membrane (BM). The $\mathrm{BM}$ is a highly specialized ECM common to many types of tissues providing spatial organization to the cells and involved in a remarkable number of physiological and pathological processes, such as cell adhesion, migration, development, wound healing and cancer progression (Timpl and Brown, 1996; Charonis et al., 2005; Brown et al., 2006; LeBleu et al., 2007; Khoshnoodi et al., 2008); in addition, it serves as a reservoir for growth factors and enzymes and is responsible for the molecular sieving (Timpl and Brown, 1996). The BM is a fine (approximately 100-300 nm thick) structure that may be considered as two dimensional (2D) in respect to the range of cell size. Nowadays, tissue engineering strives to mimic the three dimensional organization of ECM with scaffolds that support cellular response and regeneration (Daley et al., 2008). However, the development of materials and surfaces that resemble the $2 \mathrm{D}$ structure of $\mathrm{BM}$ is also a challenging task; moreover, the cells often meet such environments in contact with implanted bioengineered devices. An example is the engineered vascular tissue. To date, blood contacting devices including small diameter vascular grafts, stents, hard valves, etc, suffer from a common defect - the lack of significant endothelial cells ingrowth - presumably caused by the absence of the specialized BM, resulting in an accelerated device failure (Keresztes et al., 2006). In this respect, the molecular assembly of Col IV at different materials interface gains a distinct bioengineering interest (Hudson et al., 1993; Keresztes et al., 2006) as it can be critical for the successful interaction with $\mathrm{EC}$ - a fact that should be considered to mimic the natural organization of vessel wall. 
The supramolecular structure of Col IV was extensively studied during the last two decades (Timpl and Brown, 1996; Gelse et al., 2003; White et al., 2004; Charonis et al., 2005; Brown et al., 2006; LeBleu et al., 2007; Khoshnoodi et al., 2008). Once secreted, the triple-helical heterotrimeric molecules of Col IV self-associate to form a 2D network which serves as molecular scaffold for other BM components, such as laminin, perlecans and proteoglycans (Timpl and Brown, 1996; Brown et al., 2006). Detailed in situ analysis of high resolution electron micrographs revealed that Col IV molecules self-assemble in the BM forming polygonal networks held together by overlapping and lateral interactions along the triple-helical domain and the $\mathrm{N}$ - and C-terminal end-domains ( Timpl and Brown, 1996; Charonis et al., 2005).

Like other ECM proteins Col IV is recognized by the cells via integrins - a family of cell surface receptors that provide trans-membrane links between the ECM and the cytoskeleton (Hynes, 2002; White et al., 2004). Out of the 24 integrin heterodimers $\alpha 1 \beta 1, \alpha 2 \beta 1, \alpha 10 \beta 1$, and $\alpha 11 \beta 1$ act as primary receptors for collagens ( Vandenberg et al., 1991; Kern et al., 1993; Kapyla et al., 2000; Hynes, 2002; White et al., 2004; Popova et al., 2007), but most abundantly expressed are $\alpha 1 \beta 1$ and $\alpha 2 \beta 1$ (White et al., 2004; Khoshnoodi et al., 2008). When integrins are occupied they cluster in focal adhesion complexes where specific bidirectional integrin signaling converges with other molecular pathways (Hynes, 2002). Depending on the conformation of adsorbed protein layer, however, different integrin activity may be expected (Grinnell and Feld, 1982; Kapyla et al., 2000; Keresztes et al., 2006; Ludwig et al., 2006).

Despite the extensive research on the biochemistry and physiology of Col IV (Hudson et al., 1993; Gelse et al., 2003; Keresztes et al., 2006) and its involvement in a number of human disorders (Gelse et al., 2003; Charonis et al., 2005), surprisingly little is known about the behavior of Col IV at the biomaterials interface, which in turn, determines the successful cellular interaction.

To learn more about the biological performance of $\mathrm{Col}$ IV at the biomaterial interface we followed its adsorption profile and molecular organization of the adsorbed protein layer on model hydrophilic and hydrophobic surfaces known to strongly influence the activity of other proteins (Grinnell and Feld, 1982; Tamada and Ikada, 1994; Altankov et al., 1996; Altankov and Groth, 1996; Altankov et al., 1997; Kowalczynska et al., 2005). Atomic force microscopic (AFM) studies revealed a fine near molecular size network arrangement of Col IV on hydrophilic glass which turns into a relatively thicker - growing in size polygonal network on hydrophobic ODS consisting of molecular aggregates. We further compared the biological activity of these surface-induced differently assembled Col IV layers following the interaction with human umbilical vein endothelial cells (HUVEC). We found that cells attach less efficiently on hydrophobic ODS, while the fine Col IV network on hydrophilic substrata support HUVEC interaction involving both $\alpha 1$ and $\alpha 2$ integrins. Details of this study are presented below.

\section{Material and Methods}

\section{Preparation of hydrophilic and hydrophobic surfaces} To render the surface hydrophilic, glass coverslips $(22 \times 22$ mm, Fisher Bioblock, Thermo Fisher Scientific, Waltham, MA, USA) were cleaned in an ultrasonic bath for $10 \mathrm{~min}$ in a 1:1 mixture of 2-propanol and tetrahydrofuran. The samples were then exposed to piranha solution $(30 \%$ (v/ v) $\mathrm{H}_{2} \mathrm{O}_{2}$ and $70 \%(\mathrm{v} / \mathrm{v}) \mathrm{H}_{2} \mathrm{SO}_{4}$ ) for $30 \mathrm{~min}$ followed by a copious rinsing with milliQ water $(18.2 \mathrm{M} \Omega)$ and dried.

A hydrophobic surface was prepared according to a previously described protocol (Gustavsson et al., 2008) using an organosilane trichloro-(octadecyl)-silane (ODS) purchased from Sigma (St. Louis, MO, USA) (Cat. No 104817). Before silanization the samples were pre-cleaned as above and then placed in a solution containing $12.5 \mathrm{ml}$ of carbon tetrachloride, $37.5 \mathrm{ml}$ of heptane and $220 \mu \mathrm{l}$ ODS. The samples were left in this solution for $18 \mathrm{~min}$ at room temperature and the excess of silane was washed away with pure heptane. Samples were then heated for one hour at $80^{\circ} \mathrm{C}$.

The wettability of surfaces was estimated with water contact angle measurements using sessile drop technique performed on Dataphysics Contact Angle Systems OCA15. Average values were obtained from at least ten different samples.

\section{Quantification of adsorbed FITC-Collagen IV}

The adsorption of FITC-Collagen IV was quantified by $\mathrm{NaOH}$ extraction of the protein as described previously (Gustavsson et al., 2008). Briefly, the model surfaces were cleaned with distilled water in an ultrasonic bath. The triplicate samples were dried and coated for $30 \mathrm{~min}$ at $37^{\circ} \mathrm{C}$ with DQTM Collagen type IV (Molecular Probes, Eugene, OR, USA; Cat. No D-12052) from human placenta that was fluorescein isothiocianate conjugated (FITC-Col IV) and dissolved in phospahate-buffered saline (PBS) at the indicated concentrations. After coating at $37^{\circ} \mathrm{C}$ the samples were rinsed three times with PBS and dried. The adsorbed FITC-Col IV was extracted with $250 \mu \mathrm{l}$ of $0.2 \mathrm{M} \mathrm{NaOH}$ for $2 \mathrm{~h}$ at room temperature. The fluorescent intensity of the extracts were measured with a fluorescent spectrophotometer (Horiba-Jobin Yvon, Edison, NJ, USA), set to $488 \mathrm{~nm}$ (excitation) and $530 \mathrm{~nm}$ (emission) and compared to a standard curve based on known concentrations of FITC-Col IV solutions in $0.2 \mathrm{M} \mathrm{NaOH}$.

\section{Atomic force microscopy}

We have used the AFM type NanoScope III from Digital Instruments (Santa Barbara, CA, USA) to follow the Col IV adsorption profile and the morphology of the adsorbed protein layer operating in the tapping mode in air. Si cantilevers from Veeco (Manchester, UK) were used with a force constant of $2.8 \mathrm{~N} / \mathrm{m}$ and a resonance frequency of $75 \mathrm{kHz}$. The phase signal was set to zero at the resonance frequency of the tip. The tapping frequency was $5-10 \%$ lower than the resonance frequency. Drive amplitude was $200 \mathrm{mV}$ and the amplitude set-point Asp was 1.4V. The ratio between the amplitude set-point and the free 
amplitude was kept equal to 0.7. Several AFM images were analyzed using the WSxM software (Nanotec, Madrid, Spain) to observe the topography of non coated surfaces, as well as, the typical protein distribution on the different substrata.

\section{Cells}

Human Umbilical Vein Endothelial Cells (HUVEC) purchased from PromoCell (Heidelberg, Germany; Cat No C-12200) were cultured in Endothelial Cell Growth Medium (PromoCell, Cat No C-22010) supplemented with SupplementMix (PromoCell Cat No C39215) containing $0.4 \%$ ECGS/H; $\%$ Fetal Calf Serum, $1 \mathrm{ng} / \mathrm{ml}$ Epidermal Growth Factor, $1 \mu \mathrm{g} / \mathrm{ml}$ hydrocortison and $1 \mathrm{ng} / \mathrm{ml}$ basic fibroblast factor. For the experiments the cells were detached from around confluent flasks with Trypsin/EDTA (Invitrogen, Carlsbad, CA, USA) and the remaining trypsin activity was stopped with $100 \%$ fetal bovine serum (FBS) before 2 times washing with medium without supplements. Finally the cells were counted and reconstituted in serum free EC medium.

\section{Overall cell morphology}

To study the overall cell morphology of adhering HUVEC the cells were stained for actin. For that purpose, $10^{5}$ cells/ well were seeded in 6 well TC plates (Costar, Corning, Lowell, MA, USA) containing the samples for $2 \mathrm{~h}$ in serum free medium. Typically, the samples had been pre-coated with native Col IV (Abcam, Cambridge, UK; Cat. No ab7536, ) at a concentration of $50 \mu \mathrm{g} / \mathrm{ml}$ in $0.1 \mathrm{M}$ sodium acetate $\mathrm{pH} 4.5$. At the end of incubation, the cells were fixed with $4 \%$ paraformaldehyde $(10 \mathrm{~min})$ and permeabilized with $0.5 \%$ Triton X-1000 for 5 min. Actin cytoskeleton was visualized with $20 \mu \mathrm{g} / \mathrm{ml}$ AlexaFluor 488 phalloidin (Molecular Probes, Eugene, OR; Cat No A 12379) in PBS, and finally mounted in Mowiol (Polysciences, Warrington, PA, USA. In some cases phalloidin was added to the secondary antibody solution (e.g., for vinculin staining - see below). The samples were viewed and photographed at 10x objective on a fluorescent microscope (Nikon Eclipse E800; Nikon, Tokyo, Japan) where at least 3 representative images were acquired.

\section{Quantification of cell adhesion and spreading}

Morphological parameters such as number of adhering cells and mean cell surface area were evaluated using the Image J plug-ins (NIH, Bethesda, USA; http://rsb.info.nih.gov/ $\mathrm{ij} /$ ). The adhesion was measured by counting the cells in 3 randomly chosen images of actin stained samples to obtain the number of cells per $\mathrm{cm}^{2}$. Data were collected from at least 3 independent experiments and the average cell area was further measured for each individual image (in $\mu \mathrm{m}^{2}$ ), and calculated for each condition.

Statistical analysis was performed with ANOVA software using multiple comparison Fisher's test to determine statistically significant differences between groups $(p<0.05)$. Each data point represents mean \pm standard deviation (SD) for at least three independent experiments.

\section{Immunofluorescence}

Visualization of focal adhesion contacts. 1 x $10^{5}$ cells/ well were seeded as described above on native Col IV coated model surfaces for $2 \mathrm{~h}$ in serum free medium. To visualize focal adhesions fixed and permeabilized samples were saturated with $1 \%$ albumin in PBS for $15 \mathrm{~min}$. Vinculin was visualized using monoclonal anti-vinculin antibody (Sigma, Cat No V9131) dissolved in PBS-1\% albumin for $30 \mathrm{~min}$ followed Cy 3-conjugated Affini-Pure Goat Anti-Mouse IgG (H+L) (Jackson ImmunoResearch, Newmarket, Suffolk, UK, Cat. No 115-165-062) as secondary antibody. The samples were viewed and photographed in a fluorescent microscope Nikon at high magnification (100x). At least 3 representative images were acquired for each experimental condition.

Visualization of $\alpha 1$ and $\alpha 2$ integrins was performed with monoclonal anti-human integrin alpha-1 (Chemicon, Cat No MAB1973; Millipore, Billerica, MA, USA) or alpha-2 (Abcam, No Ab24697) also for $30 \mathrm{~min}$ followed by $\mathrm{Cy}^{3}$-conjugated Goat Anti-Mouse $\mathrm{IgG}(\mathrm{H}+\mathrm{L})$ (Jackson ImmunoResearch, No 115-165-062) as secondary antibody.

\section{Results}

\section{Characterization of surfaces}

The data presented in Table 1 show a significant increase of water contact angle $\left(\mathrm{WCA}^{0}\right)$ after coating the glass with ODS. Both advancing and receding $\mathrm{WCA}^{0}$ were found to increase about 4 times $(p<0.05)$ on ODS confirming the strongly hydrophobic nature of this surface. At the same time an approximately doubled average roughness was measured by AFM on plain ODS in comparison to glass (both substrata non-coated with protein), as shown in Table 2 (left column "Plain"), confirming the homogenous coating of the surface with the silane.

\section{Quantification of adsorbed FITC-Collagen IV}

FITC-Col IV adsorption from solutions with different concentration was determined by comparison of extracted fluorescence signals to a standard curve with known FITCCol IV concentrations (see Methods section). Detectable values were obtained for each concentration (Fig. 1). Both substrata show typical saturation curves at approximate concentrations of $50 \mu \mathrm{g} / \mathrm{ml}$. ODS surfaces, however, demonstrated significantly higher signal showing about twice more adsorbed protein for each concentration.

Table 1. Values for advancing and receding WCA of model hydrophilic and hydrophobic surfaces.

\begin{tabular}{|c|c|c|}
\hline \multirow{2}{*}{ Surface } & \multicolumn{2}{|c|}{ Water Contact Angle } \\
\cline { 2 - 3 } & Advancing & Receding \\
\hline Hydrophilic & $25.4 \pm 7.0$ & $18.6 \pm 8.0$ \\
\hline Hydrophobic ODS & $103.3 \pm 3.2$ & $96.9 \pm 6.6$ \\
\hline
\end{tabular}

The measurements were made on 10 samples in triplicates. 
Table 2. Average roughness and height of the model surfaces characterized by AFM

\begin{tabular}{|c|c|c|c|c|}
\hline \multirow{2}{*}{ Surface } & \multicolumn{2}{|c|}{ Non-coated } & \multicolumn{2}{c|}{ Col IV-coated } \\
\cline { 2 - 5 } & $\begin{array}{c}\text { Average } \\
\text { roughness }\end{array}$ & $\begin{array}{c}\text { Average } \\
\text { height }\end{array}$ & $\begin{array}{c}\text { Average } \\
\text { roughness }\end{array}$ & $\begin{array}{c}\text { Average } \\
\text { height }\end{array}$ \\
\hline Hydrophilic & $0.79 \pm 0.01$ & $2.05 \pm 1.80$ & $1.11 \pm 0.17$ & $3.93 \pm 0.32$ \\
\hline Hydrophobic ODS & $1.59 \pm 0.05$ & $5.38 \pm 0.79$ & $4.38 \pm 0.33$ & $17.88 \pm 2.80$ \\
\hline
\end{tabular}

The values were obtained with Roughness analysis tool on the WSX software using at least three scans of $1 \mu \mathrm{m}^{2}$ for each model surface.
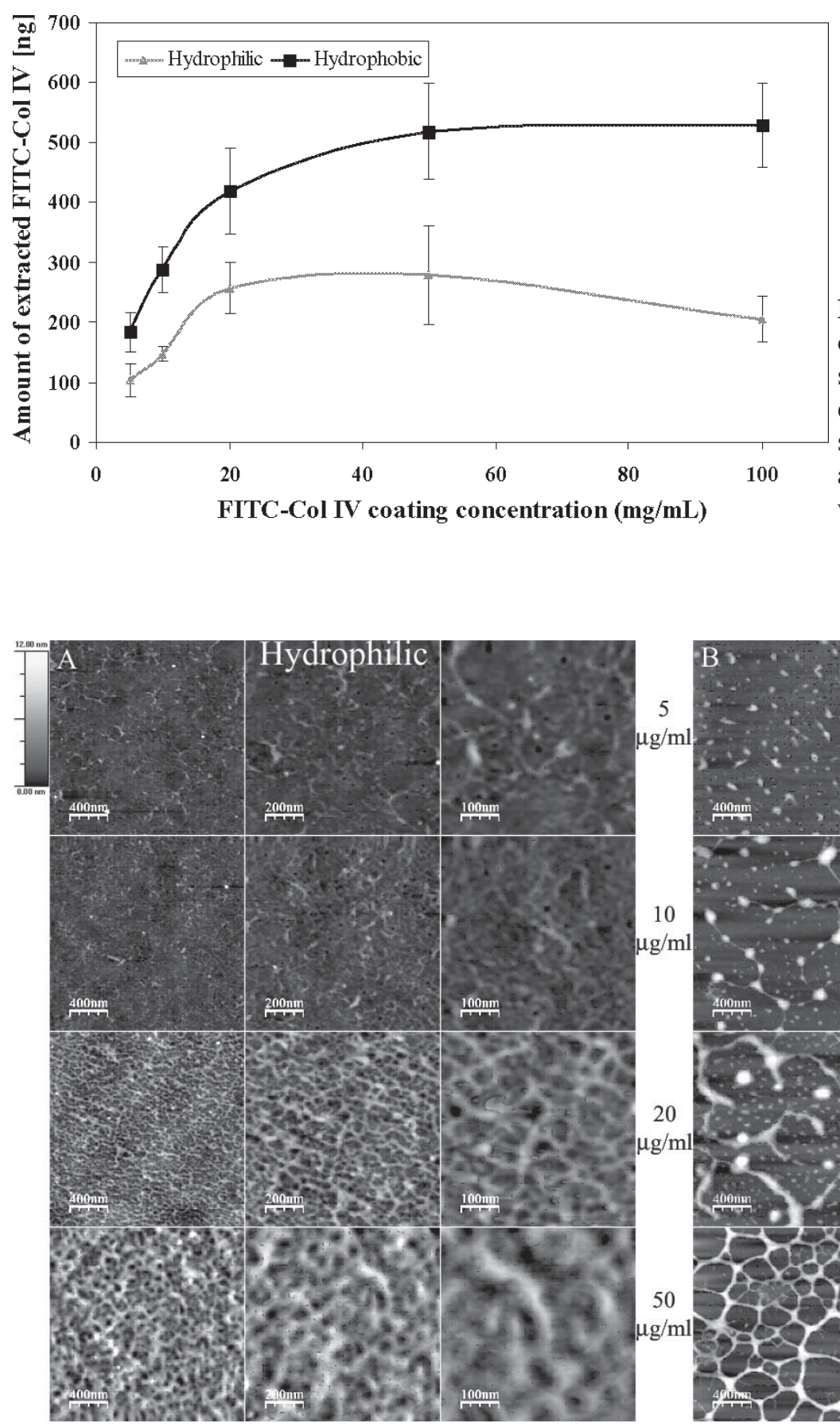

Fig. 1. Adsorption profile of FITC-Col IV on model hydrophilic and hydrophobic ODS surfaces. Triplicate measurements of extracted fluorescence were done for both substrates at different coating concentrations and the values calibrated to a standard curve with known FITC-Col IV concentration.

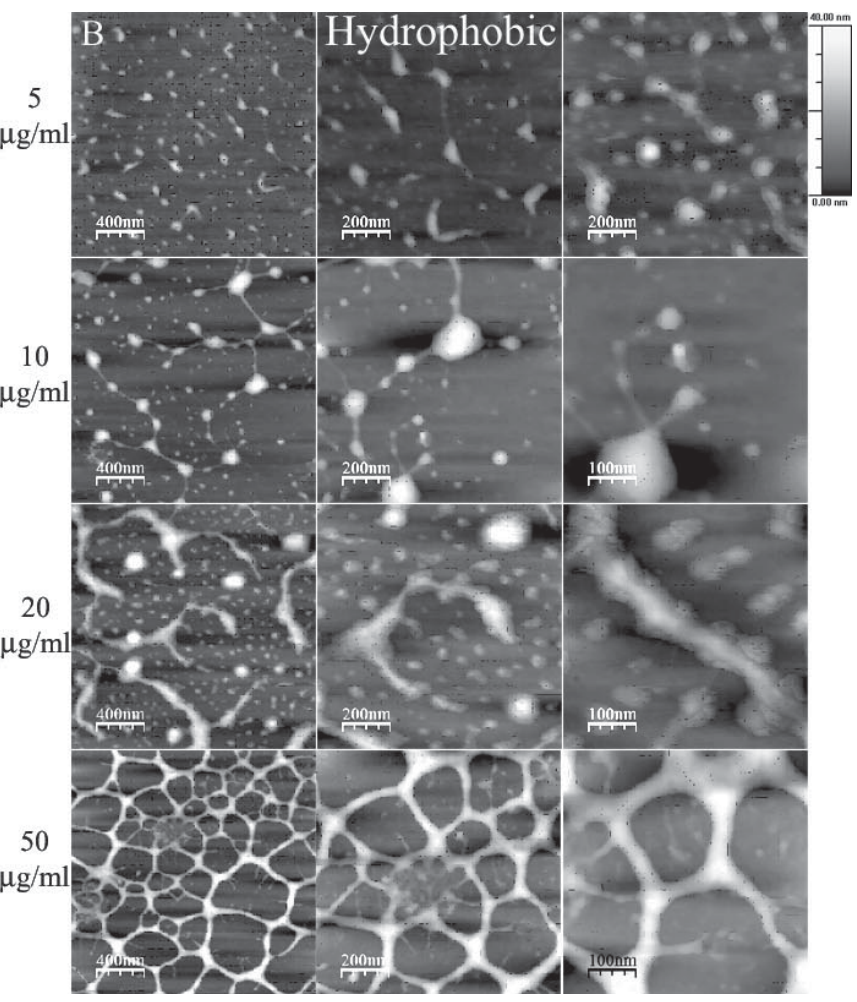

Fig. 2. AFM images of adsorbed native collagen type IV to hydrophilic (A) and hydrophobic (B) surfaces. Adsorption concentration increases from up to down and magnification increases from left to right. 

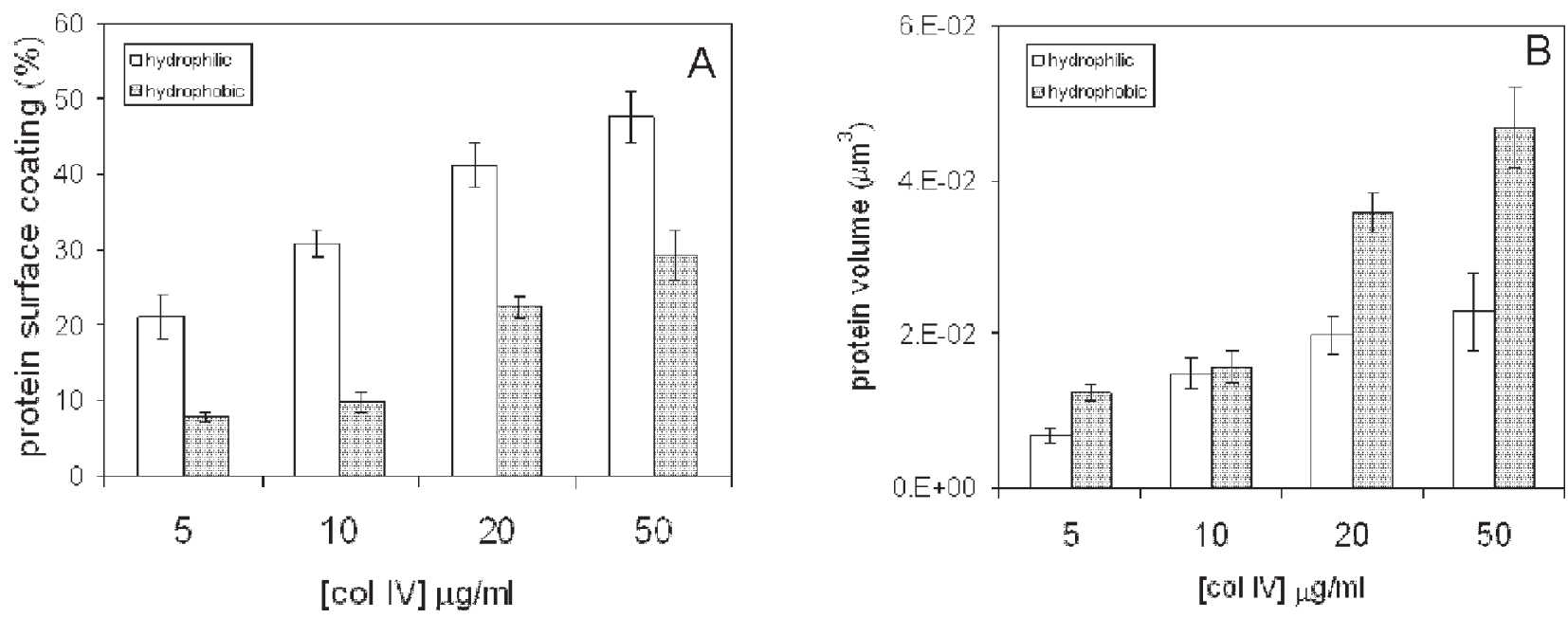

Fig. 3. Fraction of substrate covered by the protein as a function of the concentration of the initial solution (A). Collagen tends to cover larger areas on the hydrophilic than on the hydrophobic substrate. Total volume occupied by the protein on a $2 \times 2 \mathrm{~cm}^{2}$ area of the substrate (B). The total volume occupied by the protein on the hydrophobic surface is higher than on the hydrophilic one.
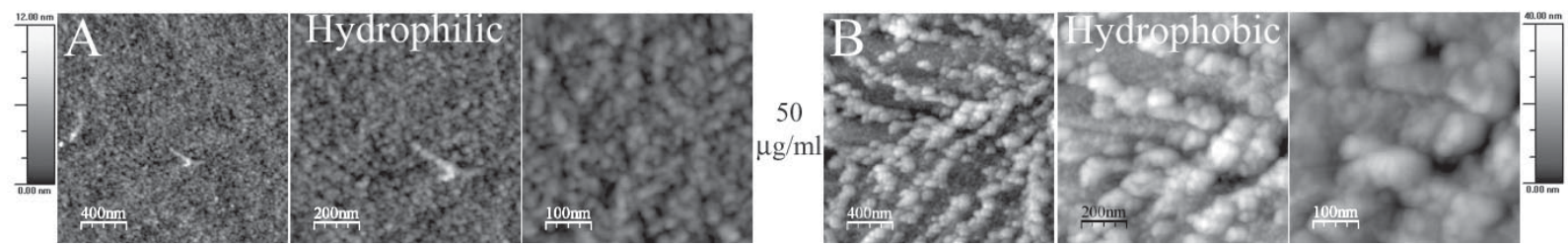

Fig. 4. AFM images of adsorbed native collagen type IV to hydrophilic (A) and hydrophobic (B) surfaces at adsorption concentration was $50 \mu \mathrm{g} / \mathrm{ml}$ for 30 minutes.

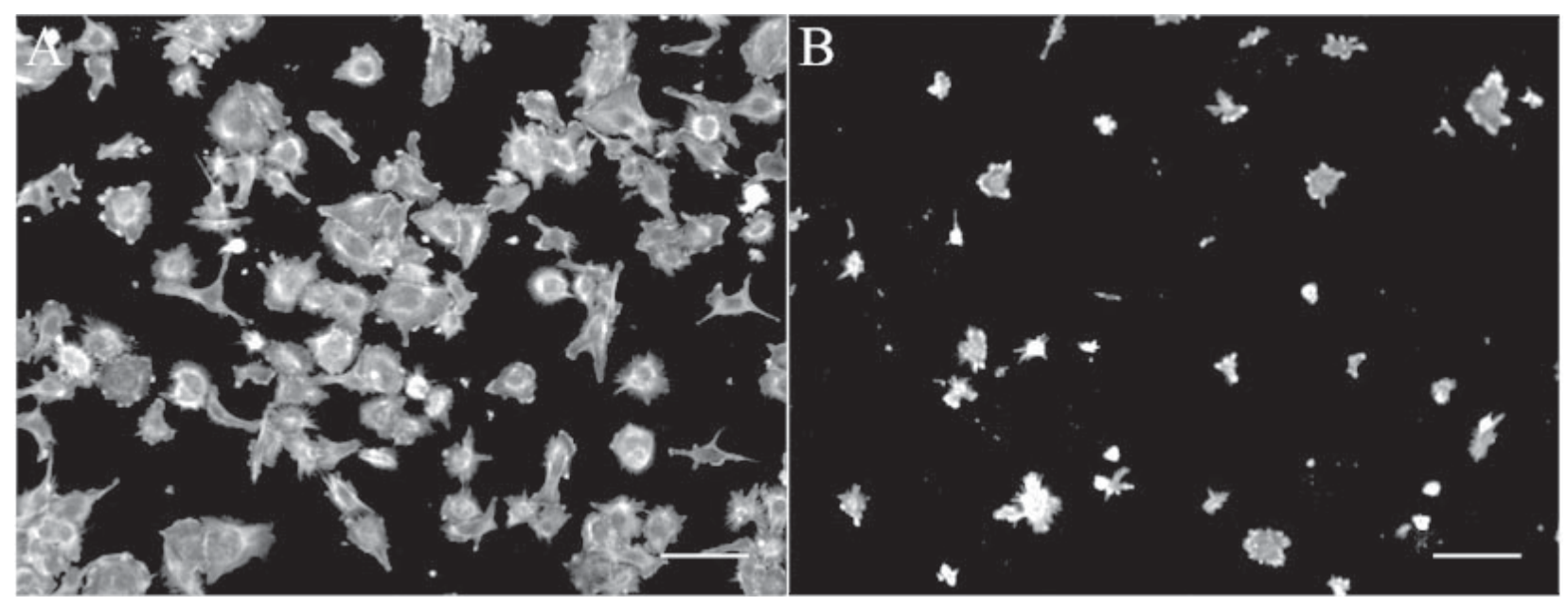

Fig. 5. Overall morphology of HUVEC adhering on native Col IV coated hydrophilic (A) and hydrophobic ODS (B) surfaces. The cells were stained for actin. Bar $=100 \mu \mathrm{m}$.

\section{Atomic force microscopy}

In the tapping mode of AFM, the cantilever oscillates with the probing tip close to its free resonance frequency at a given amplitude. The interaction between the sample and the probe gives rise to a shift in the probe vibration respective to that measured in a free oscillation, i.e., with the probe far away from the sample. The vertical displacement (height) needed to keep the set amplitude provides information about topography of the system. On the other hand, the measured phase shift may be caused by variation in the viscoelastic properties in different parts (or phases) of the sample, and in this sense it can provide some information about the morphology of the system.
However, differences in phase lag may be caused by geometric features such as edges, and can be a mere reflection of the topography of the system. There are several strategies for programming the apparatus parameters to obtain both accurate surface topographies (height) and morphologies (phase). Recent studies have shown that only when the amplitude of the vibrating cantilever is programmed to be equal to that of the free cantilever, does the height of the topography represent a true surface topography, and that a much harder tapping is necessary to observe maximum phase shift contrast between stiff and soft regions of the material (Rodriguez Hernandez et al., 2007). 

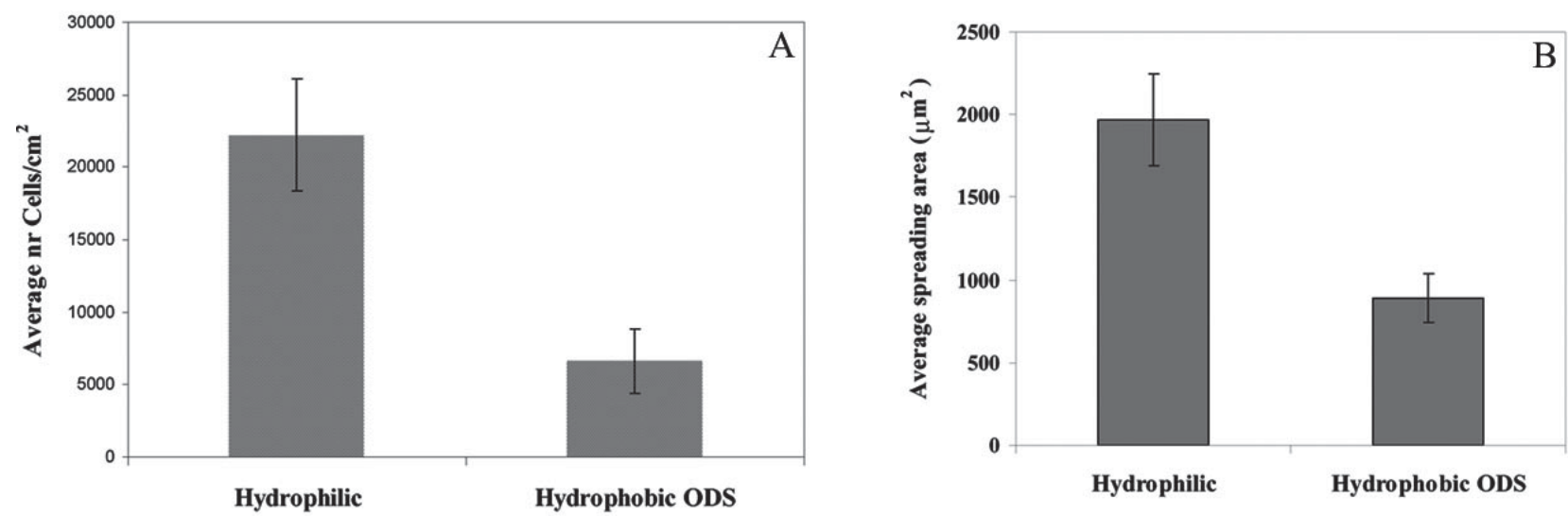

Fig. 6. Quantification of cell adhesion expressed as number of cells per $\mathrm{cm}^{2}(\mathbf{A})$ and the average spreading area measured in $\mathbf{m m}^{2} \mathbf{( B )}$ to hydrophilic and hydrophobic Col IV coated surfaces.

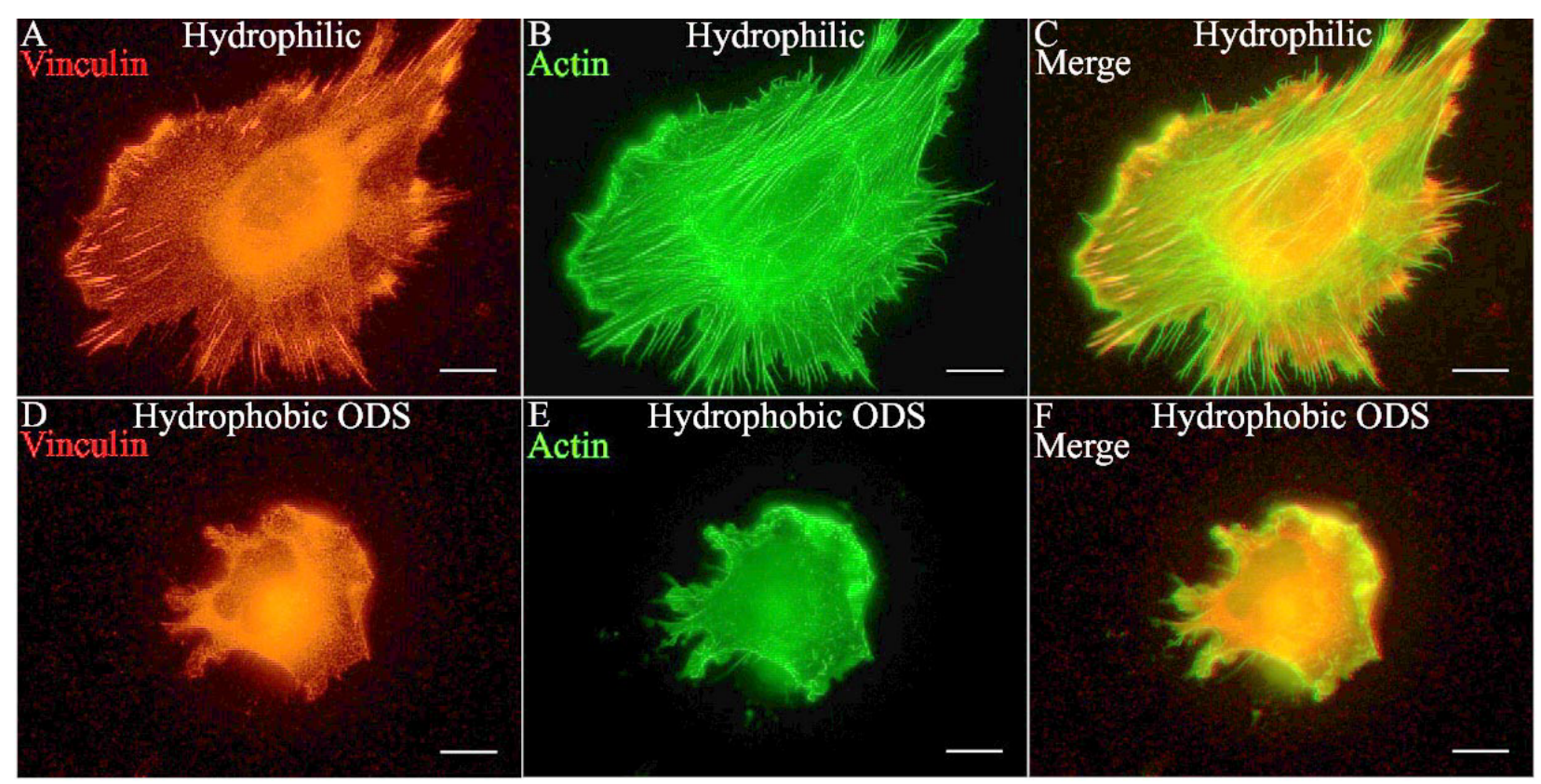

Fig. 7. Development of focal adhesions contacts and actin stress fibbers of HUVEC seeded on Col IV coated hydrophilic (A, B, C) and hydrophobic ODS (D, E, F) surfaces. Bar $=10 \mu \mathrm{m}$.

Fig. 2 shows the complex AFM images of native $\mathrm{Col}$ IV adsorbed for $10 \mathrm{~min}$ at acidic conditions $(0.1 \mathrm{M} \mathrm{Na}$ acetate, $\mathrm{pH} 4.5$ ) on hydrophilic (Fig. 2A) and hydrophobic ODS (Fig. 2B) model substrates. Overall, the organization of collagen layers changes as function of the concentrations from which the protein is adsorbed increasing as 5, 10, 20 and $50 \mu \mathrm{g} / \mathrm{ml}$ (from top to bottom). At lowest coating concentration of $5 \mu \mathrm{g} / \mathrm{ml}$, mainly single, isolated features was found on both substrates, representing elongated fibrillike morphology on hydrophilic glass (Fig. 2A) and rather globular clusters on ODS (Fig. 2B). At $10 \mu \mathrm{g} / \mathrm{ml}$ these structures enlarge on the hydrophobic substrate and tend to connect to each other (on both substrata) suggesting the initial formation of intermolecular links, i.e., the establishment of protein-protein contacts through the surface. Further increase of the coating concentrations results in the formation of networks on both surfaces, which again differ significantly in morphology. On hydrophilic glass, AFM revealed formation of a thin protein network with nearly single molecule size (see the gray scale bar) tending to cover the surface. The average height of the layers quantified using WSxM software was $3.93 \pm 0.32$ nm (see Table 2 right column entitled "Col IV coated"), which confirms the single molecule arrangement of the network. Conversely, on ODS the thickening of the observed fibril-like structures make rise to a prominent protein network (Fig. 2B, see $20 \mu \mathrm{g} / \mathrm{ml}$ and $50 \mu \mathrm{g} / \mathrm{ml}$ ) consisting of well-defined interconnected fibrils arranged in polygonal features with 300-400 nm long arms and about $20 \mathrm{~nm}$ thick. More precise WSxM measurements revealed that the average height of the layer amounted to $17.9 \pm 2.8$ nm (see Table 2, right column), which suggests the formation of supramolecular aggregates.

Considering the fact, that the non-homogenous distribution of the observed features could influence the biological activity of the protein (e.g., the accessibility to the cells) we measured the fraction of substrate covered by protein as a function of the concentration of the adsorbing protein (Fig. 3A). A significantly lower coverage of the surface was found on ODS (approx. 30\%) versus glass $(50 \%)$ at all concentrations. Nevertheless, the total volume of the adsorbed protein (Fig. 3B) was also 

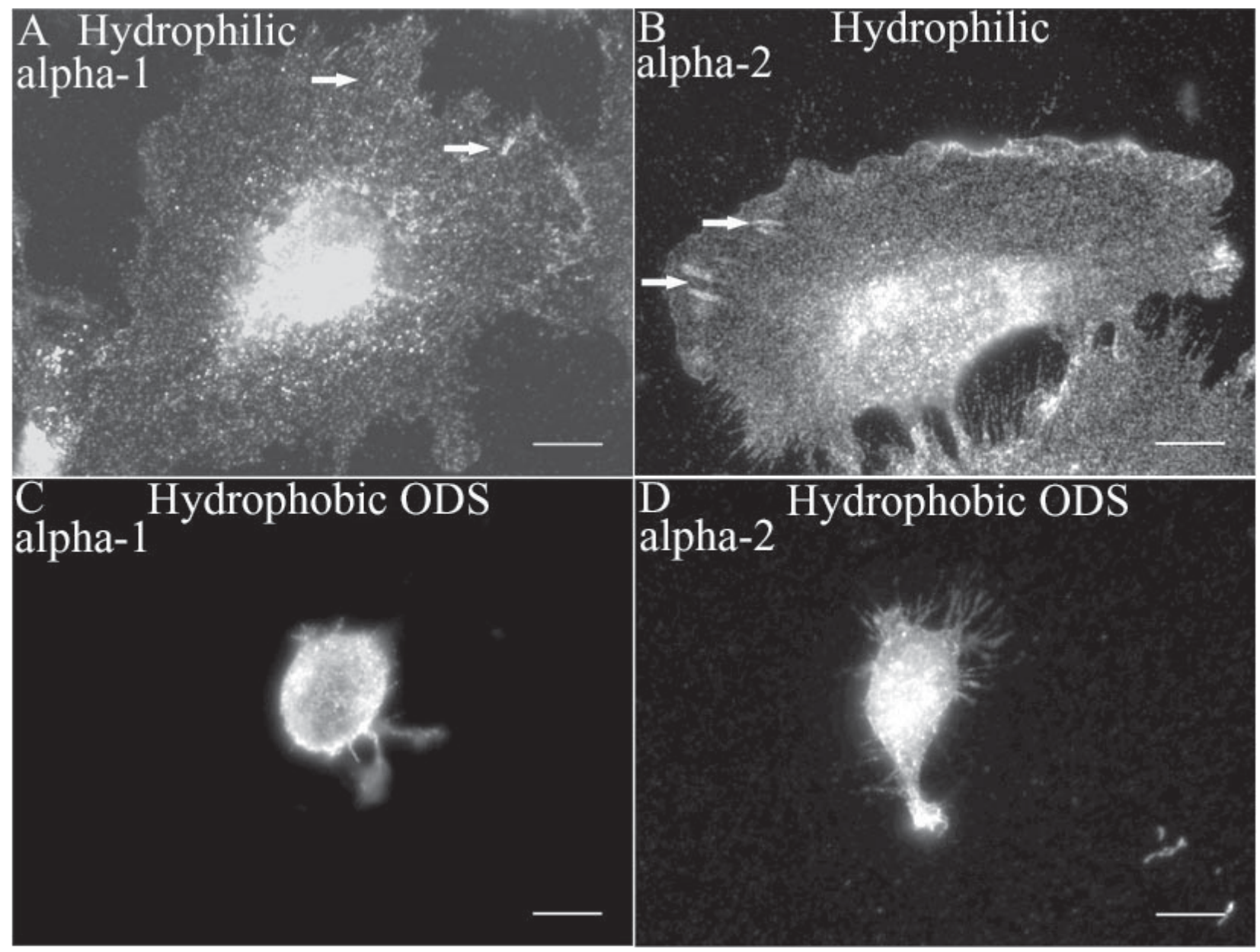

Fig. 8. Expression of alpha $1(\mathbf{A}, \mathbf{C})$ and alpha $2(\mathbf{B}, \mathbf{D})$ integrins in HUVEC adhering on hydrophilic (A, B) and hydrophobic ODS (C,D) surfaces coated with Col IV. Bar $=10 \mu \mathrm{m}$.

significantly higher on the hydrophobic surface (approximately twice) than on the hydrophilic one, confirming the same trend for different adsorption of Col IV, particularly at near saturation concentrations (Fig. 1).

For the biological studies we have used conditions for Col IV adsorption (e.g., a saturating concentration of 50 $\mu \mathrm{g} / \mathrm{ml}$ for $30 \mathrm{~min}$ at $\mathrm{pH} 4.5$ ), that have been previously established (Maneva-Radicheva et al., 2008; Tuckwell et al., 1994). The organization of the thus deposited protein layer on both hydrophilic and hydrophobic substrata is shown in Fig. 4. Overall, we found more protein bound to the surfaces although the same pattern of deposition was observed on hydrophilic glass (Fig. 4A). Predominantly multilayer-globular aggregates however appeared on hydrophobic ODS (Fig. 4B), obviously because the protein-protein interactions are favored on this surface. This results initially in a network thicker than a single molecule (seen in Fig. 2B, after $10 \mathrm{~min}$ ), giving rise to globular aggregates growing afterwards on the top (Fig. $4 \mathrm{~B}$, after $30 \mathrm{~min}$ ). Presumably the network is not seen at later times because several superimposed layers of protein are scanned.

\section{Cell adhesion and spreading}

The overall morphology of HUVEC adhering on Col IV coated hydrophilic and hydrophobic ODS substrates is shown in Fig. 5 (A and B, respectively). Generally less cell adhesion and delayed cell spreading were observed on hydrophobic ODS (Fig. 5B); the cells were round and smaller apart from hydrophilic glass where they display a typical flattened morphology (Fig. 5A). Image J software was used to quantify the morphological observations. Fig. 6 shows that both the amount of adhering cells (Fig. 6A) and the cell spreading area (Fig. 6B) differ significantly $(p<0.05)$ between Col IV coated ODS and glass samples. On later approximately 3 times more cells and with about doubled main surface area (in comparison to ODS) are found. On non-coated hydrophilic and hydrophobic surfaces only negligible adhesion and spreading were observed (not shown).

To learn more about the effectiveness of cell adhesion to Col IV the focal contacts were visualised via vinculin together with actin cytoskeleton (Fig. 7). More flattened and elongated cells on glass represented well developed focal adhesion contacts (Fig. 7A) where prominent stress fibres of the actin cytoskeleton often insert (Fig. 7B), better seen on merged images (Fig. 7C). Conversely, on hydrophobic ODS round shaped cells with almost missing focal adhesion complexes (Fig. 7D) and less developed actin cytoskeleton (Figs. 7E and 7F) were typically observed.

\section{Distribution of $\alpha 1 \alpha 2$ integrins}

To learn which integrins are involved in the adhesion of HUVEC to Col IV we studied the expression and functional organization of both $\alpha 1$ and $\alpha 2$ subunits, 
representing the most abundantly expressed collagen receptor heterodimers $\alpha 1 \beta 1$ and $\alpha 2 \beta 2$ (Hynes, 2002; Khoshnoodi et al., 2008). As shown in Fig. 8 both integrins were found to cluster pronouncedly on hydrophilic substrata (Fig. 8A and B), but $\alpha 2$ (Fig. 8B) tends to localize in the focal adhesion complexes (arrows) while $\alpha 1$ is more diffusely organized (Fig. 8A) in dot-like contacts. Conversely, the almost missing $\alpha 1$ and $\alpha 2$ integrin organization on ODS (Figs. 8C and D, respectively) correlates well with the observed absence of adhesive complexes in HUVEC on the same substratum (Fig. 7D).

\section{Discussion}

This study is consistent with the emerging field of tissue engineering aimed at reproducing the functional architecture of BM, and particularly the BM of vascular tissue. More specifically, we investigated the fate of exogenously added type IV collagen on model biomaterial surfaces to learn more about the role of substratum hydrophobicity in its biological performance. We further address the interaction with endothelial cells, considering their important role in the physiology of the vessel wall, aiming to understand their behaviour in contact with foreign materials interfaces. While EC procurement technologies for seeding blood-contacting devices have significantly improved, adhered cells often dedifferentiate or detach which accelerate device failure. We anticipate as a possible reason could be the missing environmental signals from the BM, which in turn, might be reproduced on the biomaterials interface.

Wettability has long been recognized as an important surface parameter for the materials biocompatibility (Grinnell and Feld, 1982; Altankov et al., 1997; Rodriguez Hernandez et al., 2007). In general, hydrophilic surfaces support cellular interaction, fact usually attributed to appropriate conformation of adsorbed adhesive proteins (Grinnell and Feld, 1982; Altankov et al., 1997; Kowalczynska et al., 2005; Rodriguez Hernandez et al., 2007). However, this is not always straightforward since materials with very high wettability, which bind much water like hydrogels, do not support protein adsorption and cell adhesion (Tamada and Ikada, 1994; Gugutkov et al., 2010). However, gels from natural polymers like collagen, gelatin or fibrin are good substrata for cell attachment (Brown et al., 2006; Daley et al., 2008) and work well also in adsorbed state (Tamada and Ikada, 1994; Ludwig et al., 2006). Conversely, strongly hydrophobic surfaces may support cell adhesion, for example, due to the spontaneous protein network assembly (Gurdak et al., 2006; Rodriguez Hernandez et al., 2007; Gugutkov et al., 2009; Gugutkov et al., 2010). Col IV cannot form gels as it is not fibrillar protein, but it strongly promotes cell adhesion through binding to $\alpha 1 \beta 1$ integrin via sequence localized in a spatial vicinity of Asp461 on $\alpha 1$ (IV) chain and Arg461on $\alpha 2$ (IV) chain (Tuckwell et al., 1994), or via $\alpha 2 \beta 1$ integrins which recognize the GFOGER motif in the $\alpha$ I domain (Knight et al., 1998; Zhang et al., 2003). The role of substratum wettability for Col IV assembly and its subsequent biological recognition is not clear, although some recent studies describe the surface arrangement of fibrillar type I collagen (Dupont-Gillain et al., 2005; Gurdak et al., 2006; Keresztes et al., 2006). Our results show that adsorption of FITC-Col IV is strongly dependent on substratum wettability. It reaches saturation at approximately $50 \mu \mathrm{g} / \mathrm{ml}$ coating concentration, for both kind of surfaces, although the hydrophobic ODS represents significantly higher adsorption, approximately twice in comparison to glass. Further increase of coating concentration or increasing the coating time lead to the additional formation of molecular aggregates as we found for ODS (Fig. 4) which also increased the extracted fluorescence signal with about $30 \%$ at concentration 200 $\mu \mathrm{g} / \mathrm{ml}$ (not shown). This result is not surprising since many proteins represent stronger adsorption to hydrophobic substrata due to the polar interactions (Grinnell and Feld, 1982), although, this is often not in correlation with their biological activity. As labeling of Col IV with FITC could interfere its native configuration, we studied the adsorption pattern of native (acid soluble) Col IV with AFM. The results confirmed the above difference as about twice higher volume of adsorbed protein was found on ODS, but also showed that Col IV tends to arrange in a different pattern: a fine near single molecular network on hydrophilic substrata which turn to much thicker polygonal network arrangements on hydrophobic ODS. This fact suggests that Col IV has different affinity depending on the substratum hydrophobicity. As Col IV molecules arrives to the surface, adsorption takes place on the hydrophilic substrate up to forming a single monolayer of protein that maintains even after long-term adsorption (Fig. 4) suggesting a conformation of the protein with diminished inter-molecular interaction; whereas, on the hydrophobic ODS, once a few molecules are adsorbed on the surface, the newly arriving ones from the solution tend to form multilayer aggregates rather than occupying empty space on the substrate. This points to the possibility that the molecular conformation of the protein is different on each surface, since only ODS favors the formation of multiple protein layers. Moreover, it is suggested that protein-protein contacts are favored on the hydrophobic ODS which results, first in a thicker than molecular size network (Fig. 2) and afterwards - in the long term - giving rise to multilayer-adsorption of the protein in the form of aggregates after the substrate's surface is covered (Fig. 4B).

Type IV collagen molecules are heterotrimers of about $390 \mathrm{~nm}$ long composed of three alpha chains existing in six genetically distinct forms. In vivo these isoforms organize into a unique network that provides BM specificity (Hudson et al., 1993). Assembly of type IV collagen is initiated by the formation of protomers (Timpl et al., 1985; Siebold et al., 1988) where three alpha chains associate through their non-collagenous domains before folding into a triple helices (Timpl et al., 1985; Hudson et al., 1993). The lateral association of the triple helix, the covalent binding of 7S domains and the association of alpha chains at the $\mathrm{NC} 1$ domains are essential for the formation of the protomeric network that serves as a 
scaffold for BM constituents. AFM studies revealed spontaneous in vitro assembly of Col IV in di- and tetramers upon adsorption to mica (Chen and Hansma, 2000), which suggest a similar assembly of Col IV in our conditions. At least it is the case at the hydrophilic interface, where both globular and linear fibril-like features are visible at low coating concentration (Fig.2A upper row). But the study also showed that at higher concentration, Col IV molecules assemble in a fine network that tends to cover the surface. The size of these linear structures is in the range of 300-400 nm in length and of about 3-4 nm thick suggesting a single molecule arrangement.

Another novel observation in this study is that on hydrophobic environment Col IV assemble in a completely different way. Depending on the concentration it may represents single globular aggregates, which tend to connect over the substratum as the concentration increases. Thus at coating concentration of $50 \mu \mathrm{g} / \mathrm{ml}$ these aggregates already form a well-established network where globular features disappear on early stages of adsorption. This suggests a non-physiological molecular arrangement. Moreover, the average height of the layer is $17.9 \pm 2.8 \mathrm{~nm}$ (see Table 2) confirming the formation of supra molecular aggregates which obviously become globular at longer adsorption time (Fig. 4). Interestingly, although the amount of adsorbed protein is about twice as high on hydrophobic ODS, obtained by both extracted fluorescence and AFM approaches, the ratio of the occupied surface area on AFM images is significantly less, i.e. about $30 \%$ from the total substratum area is covered in comparison to $50 \%$ for glass - a fact again suggesting stronger intermolecular interaction.

An important question is how the observed difference in the organization of self-assembled Col IV layer affects its biological performance. It is well documented that protein adsorption depends on the surface properties which produces great impact on the cellular interaction (Altankov et al., 2000; Keselowsky et al., 2003; Lan et al., 2005). Here we found that significantly increased Col IV adsorption on hydrophobic ODS does not support cellular interaction, a fact observed also for other matrix proteins (Grinnell and Feld, 1982; Tzoneva et al., 2002) and cell systems (Grinnell and Feld, 1982). The initial adhesion of HUVEC was strongly altered on ODS accompanied with delayed cell spreading and nearly absent focal adhesion complexes. This point to the possibility that aggregated conformation of adsorbed Col IV is less recognizable for the cells, which correlates with the altered development of actin cytoskeleton and integrin clustering. That is to say, the different patterns observed by AFM must be linked to the reduced availability of the binding site for both $\alpha 1$ and $\alpha 2$ integrins located most probably approximately 100 $\mathrm{nm}$ away from the amino-terminus within cyanogen bromide fragment CB3 (Timpl et al., 1985; Hudson et al., 1993; Khoshnoodi et al., 2008). But even in the case that adsorption of Col IV on hydrophobic substrates took place in a conformation that allowed adequate CB3 exposition its density would not be enough since most of the domains must be hidden due to lateral interactions between chains. This is obviously not the case on hydrophilic substrata because of the single molecule distribution of adsorbed Col IV layer (Fig. 2A). We found also higher accumulation of $\alpha 1$ and $\alpha 2$ integrins on hydrophilic glass and despite the observation that $\alpha 2$ tend to localize in focal adhesion complexes while $\alpha 1$ is more diffusely organized, this suggests stronger involvement of both $\alpha 1 \beta 1$ and $\alpha 1 \beta 2$ collagen receptors in EC spreading under these conditions. Although data exist for predominant recognition of Col IV by $\alpha 1 \beta 1$ integrin (Gardner et al., 1996) other authors state that Col IV express additionally two binding sites for o2 integrins (Kern et al., 1993) which could well explain the observed agonistic expression of $\alpha 1$ and $\alpha 2$ subunits. But why they organize differently during endothelial cells spreading in hydrophilic environments remains unclear. Nevertheless, both integrins looked inactive on hydrophobic surfaces as no organization was found suggesting very low specific recognition of adsorbed Col IV by HUVEC.

\section{Conclusion}

A novel observation in this study is the different substratum-induced assembly of Col IV on hydrophilic and hydrophobic environment, a nearly single molecular network arrangement on hydrophilic glass and prominent polygonal network consisting of molecular aggregates on hydrophobic ODS surface. We further found that endothelial cell attach less efficiently to the aggregated form of Col IV, although twice as much adsorbed protein was observed, while the fine Col IV network on hydrophilic substrata is well recognized by the cells via both $\alpha 1$ and $\alpha 2$ integrins. Thus, the material surface wettability appears to be a powerful tool for tailoring the appropriate arrangement of Col IV on foreign materials interface, particularly applicable in vascular tissue engineering.

\section{References}

Altankov G, Grinnell F, Groth T (1996) Studies on the biocompatibility of materials: fibroblast reorganization of substratum-bound fibronectin on surfaces varying in wettability. J Biomed Mater Res 30: 385-391.

Altankov G, Groth T (1996) Fibronectin matrix formation by human fibroblasts on surfaces varying in wettability. J Biomater Sci Polymer Ed 8: 299-310.

Altankov G, Groth T, Krasteva N, Albrecht W, Paul D (1997) Morphological evidence for a different fibronectin receptor organization and function during fibroblast adhesion on hydrophilic and hydrophobic glass substrata. J Biomater Sci Polymer Ed 8: 721-740.

Altankov G, Thom V, Groth T, Jankova K, Jonsson G, Ulbricht M (2000) Modulating the biocompatibility of polymer surfaces with poly(ethylene glycol): effect of fibronectin. J Biomed Mater Res 52: 219-230.

Brown B, Lindberg K, Reing J, Stolz DB, Badylak SF (2006) The basement membrane component of biologic scaffolds derived from extracellular matrix. Tissue Eng 12: 519-526. 
Charonis A, Sideraki V, Kaltezioti V, Alberti A, Vlahakos D, Wu K, Tsilibary E (2005) Basement membrane peptides: functional considerations and biomedical applications in autoimmunity. Curr Med Chem 12: $1495-1502$.

Chen CH, Hansma HG (2000) Basement membrane macromolecules: insights from atomic force microscopy. J Struct Biol 131: 44-55.

Daley WP, Peters SB, Larsen M (2008) Extracellular matrix dynamics in development and regenerative medicine. J Cell Sci 121: 255-264.

Dupont-Gillain CC, Jacquemart I, Rouxhet PG (2005) Influence of the aggregation state in solution on the supramolecular organization of adsorbed type I collagen layers. Colloids Surf B Biointerfaces 43: 179-186.

Gardner H, Kreidberg J, Koteliansky V, Jaenisch R (1996) Deletion of integrin alphal by homologous recombination permits normal murine development but gives rise to a specific deficit in cell adhesion. Dev Biol 175: 301-313.

Gelse K, Poschl E, Aigner T (2003) Collagens structure, function, and biosynthesis. Adv Drug Deliv Rev 55: 1531-1546.

Griffith LG, Naughton G (2002) Tissue engineering current challenges and expanding opportunities. Science 295: 1009-1014.

Grinnell F, Feld MK (1982) Fibronectin adsorption on hydrophilic and hydrophobic surfaces detected by antibody binding and analyzed during cell adhesion in serumcontaining medium. J Biol Chem 257: 4888-4893.

Gugutkov D, Gonzalez-Garcia C, Rodriguez Hernandez JC, Altankov G, Salmeron-Sanchez M (2009) Biological activity of the substrate-induced fibronectin network: insight into the third dimension through electrospun fibers. Langmuir 25: 10893-10900.

Gugutkov D, Altankov G, Rodriguez Hernandez JC, Monleon Pradas M, Salmeron Sanchez M (2010) Fibronectin activity on substrates with controlled - OH density. J Biomed Mater Res A 92: 322-331.

Gurdak E, Rouxhet PG, Dupont-Gillain CC (2006) Factors and mechanisms determining the formation of fibrillar collagen structures in adsorbed phases. Colloids and surfaces.B, Biointerfaces 52: 76-88.

Gustavsson J, Altankov G, Errachid A, Samitier J, Planell JA, Engel E (2008) Surface modifications of silicon nitride for cellular biosensor applications. J Mater Sci Mater Med 19: 1839-1850.

Hudson BG, Reeders ST, Tryggvason K (1993) Type IV collagen: structure, gene organization, and role in human diseases. Molecular basis of Goodpasture and Alport syndromes and diffuse leiomyomatosis. J Biol Chem 268: 26033-26036.

Hynes RO (2002) Integrins: bidirectional, allosteric signaling machines. Cell 110: 673-687.

Kapyla J, Ivaska J, Riikonen R, Nykvist P, Pentikäinen O, Johnson M, Heino J (2000) Integrin alpha(2)I domain recognizes type I and type IV collagens by different mechanisms. J Biol Chem 275: 3348-3354.

Keresztes Z, Rouxhet PG, Remacle C, Dupont-Gillain C (2006) Supramolecular assemblies of adsorbed collagen affect the adhesion of endothelial cells. J Biomed Mater Res A 76: 223-233.

Kern A, Eble J, Golbik R, Kuhn K (1993) Interaction of type IV collagen with the isolated integrins alpha1 beta1 and alpha2 beta1. Eur J Biochem 215: 151-159.

Keselowsky BG, Collard DM, Garcia AJ (2003) Surface chemistry modulates fibronectin conformation and directs integrin binding and specificity to control cell adhesion. J Biomed Mater Res A 66: 247-259.

Khoshnoodi J, Pedchenko V, Hudson BG (2008) Mammalian collagen IV. Microsc Res Techn 71: 357-370.

Knight CG, Morton LF, Onley DJ, Peachey AR, Messent AJ, Smethurst PA, Tuckwell DS, Farndale RW, Barnes MJ (1998) Identification in collagen type I of an integrin alpha2 beta1-binding site containing an essential GER sequence. J Biol Chem 273: 33287-33294.

Kowalczynska HM, Nowak-Wyrzykowska M, Kolos R, Dobkowski J, Kaminski J (2005) Fibronectin adsorption and arrangement on copolymer surfaces and their significance in cell adhesion. J Biomed Mater Res A 72: 228-236.

Lan MA, Gersbach CA, Michael KE, Keselowsky BG, Garcia AJ (2005) Myoblast proliferation and differentiation on fibronectin-coated self assembled monolayers presenting different surface chemistries. Biomaterials 26: 4523-4531.

LeBleu VS, Macdonald B, Kalluri R (2007) Structure and function of basement membranes. Exp Biol Med 232: 1121-1129.

Ludwig NS, Yoder C, McConney M, Vargo TG, Kader KN (2006) Directed type IV collagen self-assembly on hydroxylated PTFE. J Biomed Mater Res A 78: 615-619.

Maneva-Radicheva L, Ebert U, Dimoudis N, Altankov G (2008) Fibroblast remodeling of adsorbed collagen type IV is altered in contact with cancer cells. Histology Histopathol 23: 833-842.

Popova SN, Lundgren-Akerlund E, Wiig H, Gullberg D (2007) Physiology and pathology of collagen receptors. Acta Physiol 190: 179-187.

Rodriguez Hernandez JC, Salmeron Sanchez M, Soria JM, Gomez Ribelles JL, Monleon Pradas M (2007) Substrate chemistry-dependent conformations of single laminin molecules on polymer surfaces are revealed by the phase signal of atomic force microscopy. Biophys $\mathrm{J}$ 93: 202-207.

Siebold B, Deutzmann R, Kuhn K (1988) The arrangement of intra- and intermolecular disulfide bonds in the carboxyterminal, non-collagenous aggregation and cross-linking domain of basement-membrane type IV collagen. Eur J Biochem 176: 617-624.

Sipe JD (2002) Tissue engineering and reparative medicine. Ann N Y Acad Sci 961: 1-9.

Tamada Y, Ikada Y (1994) Fibroblast growth on polymer surfaces and biosynthesis of collagen. J Biomed Mater Res 28: 783-789.

Timpl R, Brown JC (1996) Supramolecular assembly of basement membranes. BioEssays 18: 123-132.

Timpl R, Oberbaumer I, von der Mark H, Bode W, Wick G, Weber S, Engel J (1985) Structure and biology of the globular domain of basement membrane type IV collagen. Ann N Y Acad Sci 460: 58-72. 
Tuckwell DS, Ayad S, Grant ME, Takigawa M, Humphries MJ (1994) Conformation dependence of integrin-type II collagen binding. Inability of collagen peptides to support alpha 2 beta 1 binding, and mediation of adhesion to denatured collagen by a novel alpha 5 beta 1-fibronectin bridge. J Cell Sci 107: 993-1005.

Tzoneva R, Groth T, Altankov G, Paul D (2002) Remodeling of fibrinogen by endothelial cells in dependence on fibronectin matrix assembly. Effect of substratum wettability. J Mater Sci Mater Med 13: 1235 1244.

Vandenberg P, Kern A, Ries A, Luckenbill-Edds L, Mann K, Kuhn K (1991) Characterization of a type IV collagen major cell binding site with affinity to the alpha1 beta 1 and the alpha 2 beta 1 integrins. J Cell Biol 113: 1475 1483.

White DJ, Puranen S, Johnson MS, Heino J (2004) The collagen receptor subfamily of the integrins. Int $\mathrm{J}$ Biochem Cell Biol 36: 1405-1410.

Zhang WM, Kapyla J, Puranen JS, Knight CG, Tiger CF, Pentikainen OT, Johnson MS, Farndale RW, Heino J, Gullberg D (2003) alpha11 beta1 integrin recognizes the GFOGER sequence in interstitial collagens. J Biol Chem 278: $7270-7277$. 